\title{
Upfront radical neck dissection in patients cN2-3
}

\author{
José Francisco Gallegos-Hernández*, José Alberto Abrego-Vázquez, Aldo Olvera-Casas, \\ Alma Lilia Ortiz-Maldonado, Héctor Arias-Ceballos, Gerardo Gabriel Minauro-Muñoz and \\ Carolina Mesías-Andrade
}

Department of Head and Neck Tumors, Oncology Hospital, Centro Medico Nacional Siglo XXI, Instituto Mexicano del Seguro Social, Ciudad de México, Mexico

\begin{abstract}
Introduction: The standard treatment of patients with metastatic squamous cell carcinoma at loco-regionally advanced stage is the association of radiation-chemotherapy; however, a high percentage of these persist and require rescue surgery, which implies significant morbidity. Initial neck dissection could play an important role in reducing this rate of complications. Objective: To know oncological results of patients with neck metastasis. Materials and methods: Retrospective analysis of patients undergoing treatment for cervical lymph node metastasis of squamous cell carcinoma. The therapeutic sequence was decided based on volume, resectability, site and size of the primary and general condition of the patient. Overall survival was calculated based on HPV status. Results: We included 30 patients with a mean age of 57 years and a mean follow-up of 5 years: 22 men and 8 women. All with loco-regionally advanced stages, $10 \mathrm{~N} 3$ and $20 \mathrm{N2}$. In 18, determination of p16 was made in the lymph node metastases, in 13 of them it was positive and in 5, negative. Seven patients were treated with initial chemotherapy and 23 underwent neck dissection; 5-year survival was $25 \%$ and the prognosis was better in patient with positive p16. Conclusion: The treatment of cervical metastasis depends on its resectability; initial surgery should be promoted. Neo-adjuvant chemotherapy is an alternative in patients with bulky, unresectable or borderline metastases, HPV is a factor of good prognosis.
\end{abstract}

Key words: Cervical metastasis. Head and neck cancer primary unknown.

\section{Introduction}

In our setting, 60 to $70 \%$ of patients with head and neck cancer present with locoregionally advanced stag$\mathrm{es}^{1}$, either with bulky neck metastases associated with small primary tumors or large primary tumors with small cervical metastases (cN1-2 A). Patients who have multiple cervical metastases or metastases larger than $6 \mathrm{~cm}$ (cN2 and cN3) represent a therapeutic challenge. Although the association of chemotherapy and radiotherapy is the standard treatment -or radiotherapy alone in patients who are not candidates for systemic treatment-, approximately $25 \%$ of these patients have cervical tumor persistence or early recurrence and need to undergo cervical lymph node dissection (rescue). This group of patients has a higher percentage of complications after surgery ${ }^{2}$.

In patients with cervical metastases from a primary unknown squamous cell carcinoma, the therapeutic decision is not necessarily easier; $35 \%$ of them are estimated to have metastatic adenopathy characteristics that drive to expect tumor persistence after concomitant treatment (tumor necrosis, lymph node conglomerates and periganglionic tumor invasion on tomography) ${ }^{3}$.

The therapeutic sequence in patients with advanced cancer has no impact on survival, since it depends on

\section{Correspondence:}

*José Francisco Gallegos-Hernández

E-mail: gal61@prodigy.net.mx

1665-9201/@ 2019 Sociedad Mexicana de Oncología. Published by Permanyer México. This is an open access article under the terms of the CC BY-NC-ND
Date of reception: 29-11-2017

Date of acceptance: 10-05-2019 icense (http://creativecommons.org/licenses/by-nc-nd/4.0/).
Available online: 05-11-2019 Gac Mex Oncol. 2019;19:7-10 www.gamo-smeo.com
ms of the CC BY-NC-ND 
factors of the neoplasm itself such as capsular rupture, soft tissue invasion, dissected/metastatic lymph nodes ratio, etc., but it does impact on morbidity and quality of life.

Cervical metastasis standard treatment is neck dissection, and its radicalness depends on the volume of metastasis and non-ganglionic structures infiltration (sternocleidomastoid, internal jugular vein and spinal accessory nerve). This procedure allows patients to be postoperatively classified and, based on this, decide the adjuvant treatment to be followed ${ }^{4,5}$. When it is performed as a first step in combined therapy it is called "upfront dissection", and its purpose is to avoid performing the same procedure but in an area with significant anatomical changes due to concomitant treatment, which increases the risk.

However, when metastases are unresectable, combined chemotherapy and radiotherapy is the standard treatment, and surgery is performed only in case of clinical persistence or imaging evidence of residual cervical tumor on positron-emission tomography/computed tomography (PET-CT).

An alternative to initiating concomitant treatment is neoadjuvant chemotherapy in order to "make" unresectable metastases resectable and perform lymph node dissection prior to the chemoradiation association or radiation alone ${ }^{3,5}$.

When, owing to the metastatic characteristics, the patient is expected to possibly experience persistence or recurrence in the short-term, upfront dissection should be preferred in order to adequately stage the disease and reduce the rate of complications that may occur during an eventual surgical rescue ${ }^{6}$.

Human papillomavirus (HPV) status is a well-known prognostic factor, and although there is still not enough evidence to reduce treatment intensity in $\mathrm{HPV}(+)$ patients, its presence might favor the decision for upfront dissection $^{7-9}$.

We assessed the evolution of a group of patients with squamous cell carcinoma metastases to the neck, classified as cN2 and cN3, in order to know their evolution, the role of HPV in prognosis and the rate of responses to induction chemotherapy in those who were not considered candidates for upfront radical neck dissection.

\section{Material and methods}

Retrospective analysis of a series of patients consecutively treated for squamous cell carcinoma metastasis with primary tumor identified or not.
Table 1. In $53 \%$ of patients, the tumor originated in any oropharyngeal subsites, mainly in the base of the tongue; 6 patients $(20 \%)$ had an unknown primary tumor

\begin{tabular}{|l|l|l|}
\hline Tumor site & $\mathbf{n}$ & $\%$ \\
\hline Unknown primary tumor & 6 & 20 \\
\hline Tonsil & 3 & 10 \\
\hline 3-fold zone & 1 & 3.3 \\
\hline Base of the tongue & 7 & 23.3 \\
\hline Soft palate & 1 & 3.3 \\
\hline Glossotonsillar sulcus & 1 & 3.3 \\
\hline Supraglottic larynx & 1 & 3.3 \\
\hline Nasopharynx & 1 & 3.3 \\
\hline Piriform sinus & 3 & 10 \\
\hline Oropharyngeal lateral wall & 3 & 10 \\
\hline Piriform sinus & 3 & 10 \\
\hline
\end{tabular}

Clinical records of patients treated for this neoplasm for 6 consecutive years were assessed. Average follow-up was 5 years. Patients in whom the information was not available or who had not received treatment in our institution were excluded.

The type of treatment administered and the presence or absence of HPV, was determined by immunohistochemistry with $\mathrm{p} 16$ in cervical lymph nodes, and overall survival of the series and based on HPV status was calculated.

\section{Results}

The records of 30 consecutively-treated patients, 22 men and 8 women, with a mean age of 57 years, were evaluated; 20 were classified as N2 and 10 as N3.

In 18 patients, p16 analysis could be performed on cervical lymph node samples, whereas in 12 it could not be performed and the HPV status remained unknown. In 13 out of 18 patients (72\%), HPV status was positive, in $28 \%$ it was negative and in $40 \%$, HPV infection status remained unknown because testing was not performed.

The most common site of primary tumor location was the oropharynx in 17 patients $(56 \%)$, followed by unknown primary tumor in $6(20 \%)$. The location distribution of can be seen in table 1 .

On initial assessment, 23 patients were candidates for surgery and underwent radical neck dissection. There was no perioperative mortality or major complications in this group of patients, among which 7 were 
considered unresectable and received 2 cycles of induction chemotherapy, with 6 of them showing a clinical response after chemotherapy, which allowed radical neck dissection; in all of them, there was residual tumor, and no patient (0/6) had major complications after surgery. One patient showed unresectable clinical persistence after chemotherapy, and thus he received another chemotherapy cycle associated with radical radiotherapy, but since he continued showing persistence after this treatment, he had to undergo rescue surgery. As postoperative complications, he experienced hematoma, bleeding and surgical wound dehiscence that required reoperation on two occasions.

Overall survival for the series was $25 \%$, with HPV-positive patients showing a better prognosis with a 5 -year survival of $45 \%$, which is significant when compared with patients with negative or unknown HPV status (Figs. 1 and 2).

\section{Discussion}

Patients with multiple or bulky neck metastases and with unknown primary tumors receive radical radiotherapy or concomitant chemotherapy and radiotherapy as standard treatment, depending on the stage of the disease and the subsite the tumor originates from; however, approximately $25 \%$ of these patients are estimated to show persistence in spite of treatment or to have early recurrence and require rescue surgery. Rescue or salvage surgery after concomitant treatment failure is associated with a high rate of postoperative complications $^{10}$, and therefore it is preferable to perform lymph node dissection first in order to reduce morbidity. This is called "upfront neck dissection", which has the virtue of offering adequate staging and a lower rate of complications in comparison with rescue dissection ${ }^{11}$.

However, there is a group of patients who cannot undergo upfront dissection. It is those who have large tumor volumes at primary site, or cervical adenopathies regarded as unresectable. This group of patients has two therapeutic alternatives: concomitant chemotherapy-radiotherapy or neoadjuvant chemotherapy. If there is sufficient response, lymph node dissection is performed, otherwise, the treatment continues with radiotherapy or chemo-radiation if patient general status allows ${ }^{12}$.

Finally, the therapeutic goal is an adequate selection of patients that allows offering a balance between cancer control and morbidity.

There are clinical and imaging factors that predict the persistence of lymph node metastases after non-surgical treatment. These are the presence of multiple lymph

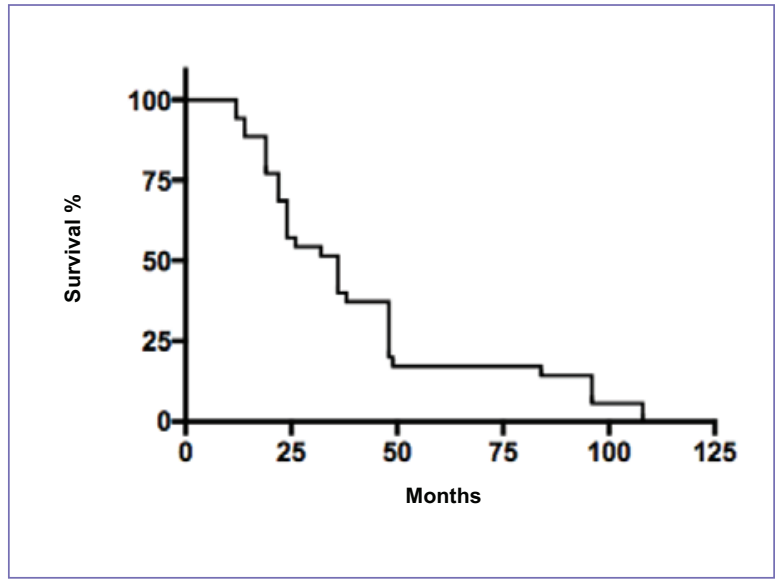

Figure 1. Five-year survival for the entire patient sample was $25 \%$, owing to the advanced stage of disease at which the diagnosis was established.

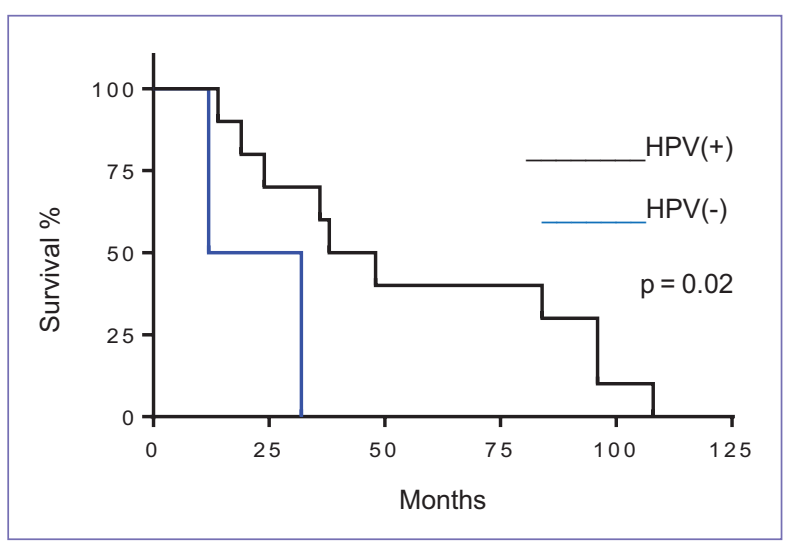

Figure 2. The presence of human papillomavirus (HPV) at metastatic lymph nodes is a factor of good prognosis when compared to HPV-negative patients.

nodes, the formation of lymph node conglomerates and the presence of lymph node tumor necrosis on cervical tomography. These lymph node characteristics indicate that upfront surgical treatment is more indicated.

Neoadjuvant chemotherapy is an alternative, although still controversial, that is valid for patients who are not candidates for upfront surgery for any reason. In this series, we found an important response to neoadjuvant chemotherapy treatment (85\%), which allowed subsequent surgery. Although neither locoregional control nor survival change with the therapeutic sequence, the rate of complications is different. In this series, there were no major complications in the group of patients who underwent upfront surgery or in those who had received neoadjuvant chemotherapy. 
HPV status is another important factor to be taken into account, since it not only impacts on patient prognosis, but could, in the immediate future, select the type of treatment. Due to the good prognosis HPV-positive patients who do not consume alcohol/tobacco have, their treatment could be less radical, avoiding, for example, chemoradiotherapy after surgery or limiting the radiotherapy fields to the oropharyngeal half without including the entire mucosa of the head and neck area ${ }^{13}$.

In the present series, survival in HPV-positive patients was significantly better when compared to patients with negative and unknown HPV status.

In the initial diagnostic-therapeutic approach of a patient with head and neck cancer, we must take into account that, in the presence of recurrence after radiotherapy, either alone or in combination with chemotherapy, as well as after bio-radiotherapy, surgical rescue is much more complex and susceptible to higher morbidity. Complications reported by other authors are significantly more serious: the rate of wound infections, flap necrosis and vascular surgery-associated injury is increased, and thus we should be very cautious both when deciding on initial treatment and when deciding if a patient is candidate for surgical rescue or not. The multidisciplinary approach used in specialized centers is essential to minimize the complication rate of this procedure ${ }^{14,15}$.

Recently, the guidelines for the management of the neck in oral and oropharyngeal cavity cancer, taking into account the morbidity rescue surgery implies, have shown evidence-based criteria for neck dissection in patients previously treated with radiotherapy or chemoradiotherapy. The rationale for the decision includes clinical evaluation, magnetic resonance and tomography imaging, size of the probable recurrence and uptake intensity on PET-CT ${ }^{16}$.

\section{Conclusions}

The treatment of patients with head and neck squamous cell carcinoma with metastasis to the lymph nodes depends on the stage, lymph node resectability, patient status and anatomical subsite of tumor origin. Balance must be achieved between cancer control and complications. The therapeutic sequence does not impact on survival, but it does on the sequels left by treatment ${ }^{17-19}$.

Upfront radical neck dissection is safe and decreases the possibility of subsequent surgery being required and for complications to increase. In patients with unresectable metastases, standard treatment is concomitant chemoradiation; however, neoadjuvant chemotherapy has emerged as an alternative for the performance of surgery after treatment in patients with sufficient response and to avoid carrying it out as rescue at the expense of higher morbidity.

In positive-HPV patients, de-escalation in therapeutic intensity and radicalness could be a reality in the near future due to the good prognosis these patients have when they are not big consumers of tobacco or alcohol ${ }^{20}$.

\section{References}

1. Gallegos-Hernández JF. Cervical lymph node metastases of squamous cell carcinoma from unknown primary. Current diagnostic and therapeutic approach. An Orl Mex. 2016;61:57-65.

2. Gallegos-Hernández JF. El cáncer de cabeza y cuello. Factores de riesgo y prevención. Cir Ciruj. 2006;74:287-93.

3. Elicin O, Albrecht T, Haynes AG, Bojaxhiu B, Nisa L, Caversaccio M, et al. Outcomes in advanced head and neck cancer treated with up-front neck dissection prior to (chemo)radiotherapy. Otolaryngol Head Neck Surg. 2016;154:300-8.

4. Sanabria A, Silver CE, Olsen KD, Medina JE, Hamoir M, Paleri V, et al. Is selective neck dissection indicated during salvage surgery for head and neck squamous cell carcinoma? Eur Arch Otolaryngol. 2014;271:3111-9.

5. Paleri V, Urbano TG, Mejhanna H, Repanos C, Lancaster J, Patel M, et al. Management of neck metastases in head and neck cancer: United Kingdom National multidisciplinary guidelines. J Laryngol Otol. 2016; 130(S2):S161-9.

6. Strojan P, Ferlito A, Langendijk JA, Corry J, Woolgar JA, Rinaldo A, et al. Contemporary management of lymph node metastases from an unknown primary to the neck: a review of therapeutic options. Head Neck. 2013; 35:286-93.

7. Jouhi L, Halme E, Iriala H, Saarilahti K, Koivunen P, Pukkila M, et al. Epidemiological and treatment-related factors contribute to improved outcome of oropharyngeal squamous cell carcinoma in Finland. Acta Oncol. 2018:57(4):541-51.

8. Flores-de la Torre C, Hernández-Hernández DM, Gallegos-Hernández JF. Human papilloma virus in patients with epidermoid head and neck carcinoma: a prognostic factor? Cir Cir. 2010;78:221-28.

9. Husain N, Neyaz A. Human papillomavirus assocaited head and neck squamous cell carcinoma: Controversies and new concepts. J Oral Craniofac Res. 2017;7:198-205.

10. Wopken K, Bijl HP, Langendijk JA. Prognostic factors for tube feeding dependence after curative (chemo-)radiation in head and neck cancer: A systematic review of the literature. Radiother Oncol. 2017;21:32545-8.

11. Elicin O, Nisa L, Dal Pra A, Bojaxhiu B, Caversaccio M, Schmücking M, et al. Up-front neck dissection followed by definitive (chemo)-radiotherapy in head and neck squamous cell carcinoma: Rationale, complications, toxicity rates and oncologic outcomes. A systematic review. Radiother Oncol. 2016;119:185-93.

12. Patil VM, Prabhash K, Noronha V, Joshi A, Dhumal S, Arya S, et al. Neoadjuvant chemotherapy followed by surgery in very locally advanced technically unresectable oral cavity cancers. Oral Oncol. 2014;50:1000-4.

13. Liu H, Li J, Zhou Y, Hu Q, Zeng Y, Mohammadreza MM. Human papillomavirus as a favorable prognostic factor in a subset of head and neck squamous cell carcinomas: A meta-analysis. J Med Virol. 2017;89:710-25.

14. Suzuki H, Hanai N, Nishikawa D, Fukuda Y, Hasegawa Y. Complications and surgical site infection for salvage surgery in head and neck cancer after chemoradiotherapy and bioradiotherapy. Auris Nasus Larynx. 2017; 44:596-601.

15. Pang $L$, Jeannon JP, Simo R. Minimizing complications in salvage head and neck oncological surgery following radiotherapy and chemo-radiotherapy. Curr Opin Otolaryngol Head Neck Surg. 2011;19:125-31.

16. Koyfman SA, Ismaila N, Crook D, D'Cruz A, Rodríguez CP, Sher DJ, et al. Management of the neck in squamous cell carcinoma of the oral cavity and oropharynx: ASCO clinical practice guideline. J Clin Oncol. 2019 Feb 27:JCO1801921. doi: 10.1200/JCO.18.01921. [Epub ahead of print].

17. Nevens D, Duprez F, Bonte K, Deron P, Huvenne W, Laenen A, et al. Upfront vs no upfront neck dissection in primary head and neck cancer radio(chemo)therapy: Tumor control and late toxicity. Radiother Oncol. 2017;124(2):220-4.

18. Tangthongkum M, KirtsreesakulV, Supanimitjaroenporn $P$, Leelasawwatsuk $P$. Treatment outcome of advance satagerd oral cavity cancer: concurrent chemo-radiotherapy compared with primary surgery. Eur Arch Otorhinolaryngol. 2017;274:2567-72.

19. Huguet F, Schick U, Pointreau Y. Role of induction chemotherapy in head and neck cancer. Cancer Radiother. 2017;21:510-4.

20. Mehta V, Moore-Medlin T, Flores JM, Ma X, Ekshyyan O, Nathan CO. Survival outcomes based on systematic agent used concurrently with radiation in human-papillomavirus associated oropharyngeal cancer. Oncotarget. 2017;8:7097-5. 